\title{
INFORMATION TECHNOLOGY AND COST OPTIMIZATION
}

\author{
Sayyad Arif Ulla ${ }^{1}$ \\ ${ }^{1}$ Asst Professor, Department of computer science, Govt Degree College, sindhanoor, Raichur, Karnataka -584128
}

\begin{abstract}
“An Approach to Balancing Capital Spending, Operating Efficiency, and Risk Mitigation”
\end{abstract}

Faced with a competitive business environment and shrinking budget, in order to cut costs, improve agility firms are viewing IT optimization as a high priority initiative.

Management is still a top priority. However, making meaningful cost reduction without affecting performance is easier said than done. This article discusses "a structured and pragmatic approach to zero-basing can help achieve sustainable cost reduction while protecting key activities." Information technology cost optimization, IT Budget, IT cost accounting, IT cost allocation, IT cost analysis, IT cost benchmarking, IT cost categories, IT cost cutting ,IT cost cutting ideas "Though world economies show some signs of emerging from recession, cost management remains a top priority and a key performance driver for most companies. Many businesses have weathered the downturn by addressing easily accessible cost reduction opportunities. However, making significant and sustainable cost reductions without threatening performance and competitiveness is more difficult because many companies are unable to systematically identify and analyze the full range of activities

\section{INTRODUCTION}

With the relentless downturn in the global economy, business growth has ground to a halt, with several companies and entire industries facing top-line declines. As a result, businesses are forced to focus on trimming their bottom lines by employing aggressive cost-cutting measures, sometimes to the detriment of strategic business transformational efforts.

In the current market scenario, optimizing services to save costs is no longer "good-to-have"; it is a "must-have". IT budgets in financial services firms have reduced and the focus of IT organizations has, in many cases, shifted to "keeping the lights on".

In such a scenario, focusing on IT optimization initiatives will not only help organizations utilize their budgets in an optimal manner, it will also bring in much needed additional savings. Many companies will find it beneficial to turn to service providers that specialize in cost optimization. But the key imperatives in these economic times are speed, results and an understanding that strategic initiatives should not be sacrificed, no matter how deep the cuts must be.

\section{KEY FINDINGS AND CHALLENGES}

- When performing IT cost optimization, recognize that not all ideas are worth the effort.

- Too often, cost optimization ideas are evaluated only on their potential to reduce costs.

- Cost optimization outside of IT requires buy-in and support from the business; a lack of such support will undermine the best efforts.

- Expending time and energy on cost optimization efforts that the organization will not embrace wastes time and undermines the credibility of the IT leadership.

\section{IT OPTIMIZATION MODELS AND TECHNIQUES}

\subsection{Identifying, Quantifying Scope and Opportunities}

As a step towards achieving the cost optimization in IT, there is requirement to identify the relevant scope and opportunity, quantifying them based on different parameters and using different methodologies. Like,

- Conduct executive-level discussions to understand priorities.

- Identify key stakeholders.

- Ensure comprehensive scope.

- Based on responses, list all possible opportunities within areas in scope. Categorize opportunities based on financial and organizational impact.

- Validate opportunities with stakeholders and executive committee. Based on the financial data, quantify the benefits from the opportunities (impact level).

- Assess their IT budgets and pinpoint optimization opportunities.

- Identify short- and long-term savings from IT resources and optimizations.

- Prepare a road map 
- Exploring alternate scenarios and preparing implementation roadmap

\subsection{IT Optimization Decision Framework}

Cost Optimization Requires a Decision Framework That Weighs Several Factors

Individual organizations employ their own strategies and define frame work to achieve the required optimization in information technology and achieve cost optimization.

As suggested by the Gartner, some of the key frameworks are well suited to achieve IT cost optimization generally.

Cost optimization rarely happens in isolation. There are almost always risks and consequences to reducing business costs. For example, what may seem like a relatively straightforward cut - postponing a planned project -has business consequences and risks that must be taken into account.
Recognizing that cost optimization teams usually operate on tight time frames, Gartner has suggested a concise decision framework to help prioritize cost optimization techniques. This framework considers not only the potential benefit (in terms of cash savings), but also the impact on customers, time requirements, degree of organizational and technical risk, and investment required

Basic framework may be used to weigh the benefits and risks of cost optimization initiatives. For each criterion (row),only one column (red, yellow or green) of the framework should be populated. For example, for the customer impact row, an initiative would be rated as negative, none or positive - not all three. Potential financial benefit must be measured to get a sense of whether the effort might be worthwhile. Customer impact must be assessed (though not necessarily quantified) to determine if an effort might have large financial benefits, but create ill will with customers, and thus undermine the value of moving forward. Even after using this decision framework, it may be necessary to group ideas together and map them to a grid to help determine the level of effort the business.

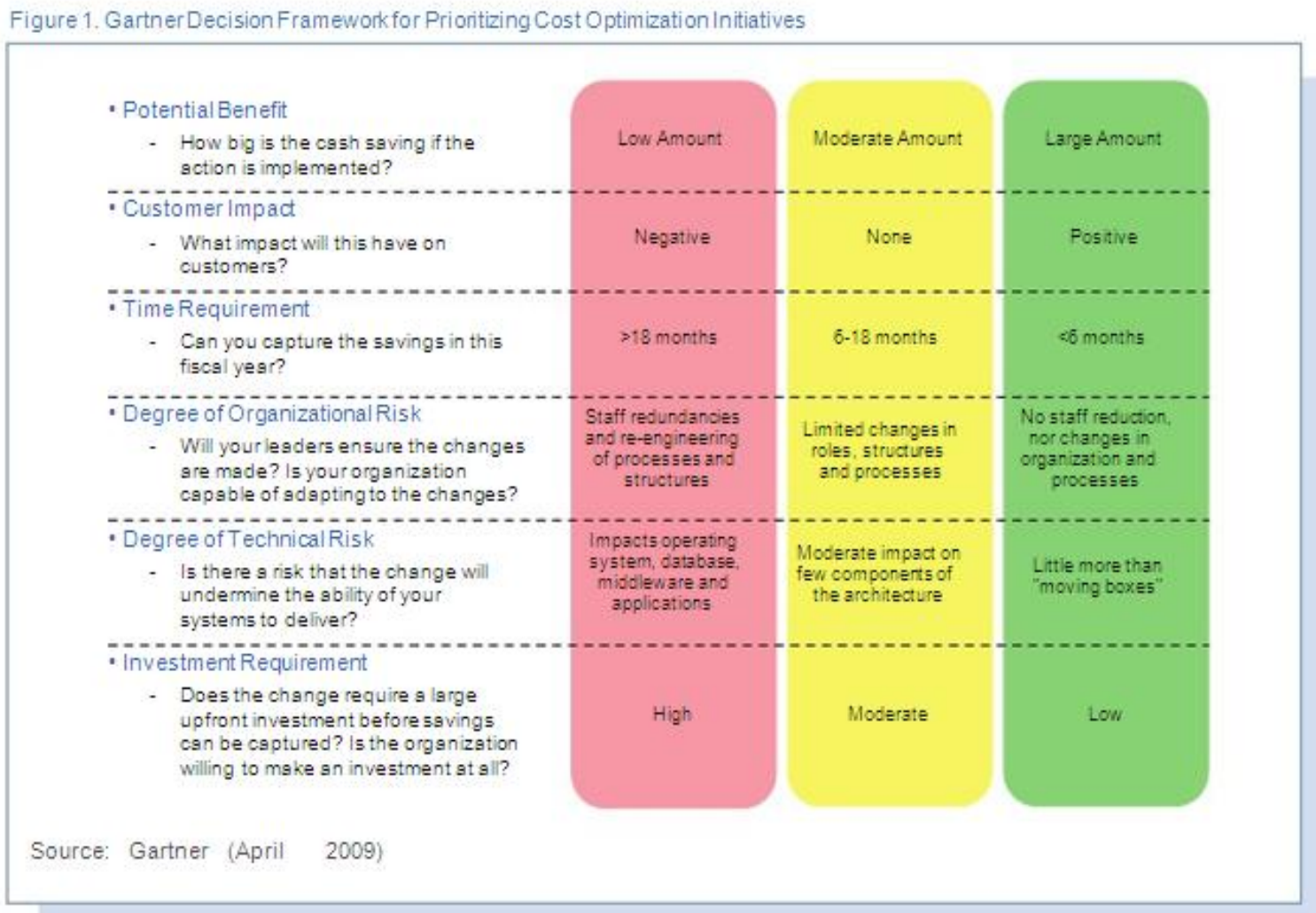


Figure 2. Grouping and Mapping Cost Optimization Techriques on a

Grid Will Help the Business See the Effort and Reward Associated With Various Techniques

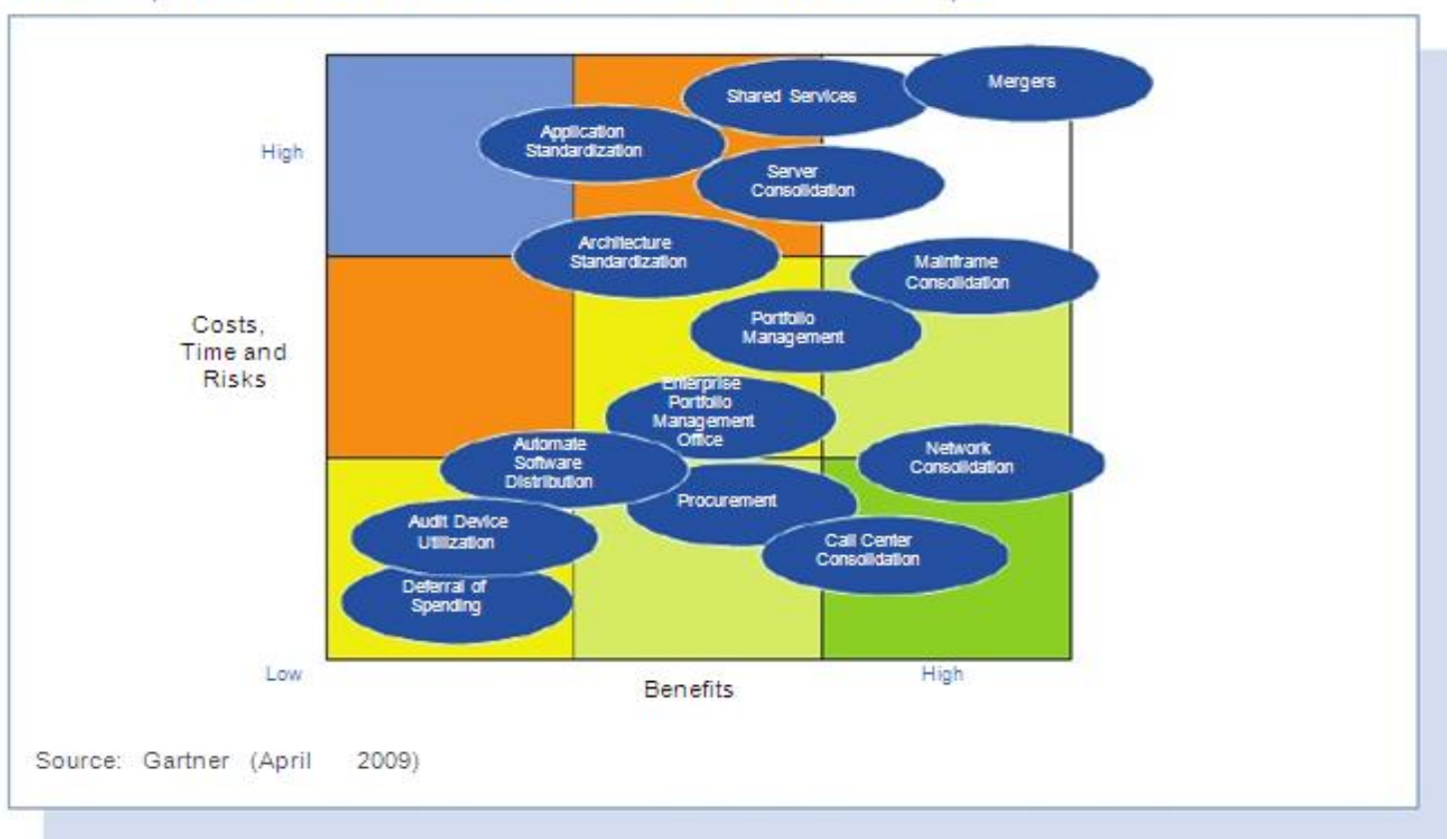

\subsection{Five Key Principles for Continuous IT Cost}

\section{Optimization}

\subsubsection{Transparency}

Higher maturity IT transparency practices are seen as a prerequisite for better supply and demand decisions, and they enable many enterprises to optimize costs and cut costs the right way, while preserving what is most important to the enterprise. IT business services must be defined and validated by the business for IT transparency and cost optimization to occur. The effectiveness of transparency needs to be considered through defining the business outcomes. This is not possible until IT and the business have agreed explicitly on what IT provides the business and what the business needs from IT. With transparency, additional benefits can include better demand management, identification of business value, and the ability to run IT like a business, better IT estimation capabilities and overall better marketing of the IT capability in general. Without transparency, the worst fears of enterprise stakeholders are often validated. With transparency, the IT organization can change its desired role in the business and enter into different value discussions that balance costs with benefits of IT-enabled endeavors and services.

\subsubsection{Agility}

One of the most significant factors on the path to continuous optimization depends on the ability of the organization to become more rapidly adaptable to internal goals and external conditions. The challenge of optimizing IT costs isn't just about trying to reduce the unit cost of IT, but trying to keep a balance between lower unit costs and sourcing IT in a flexible way so that the organization is able to remove or add resources/costs in response to any changes, from both internal and external factors.

A common problem occurs when an organization has been significantly reducing its costs, but then finds itself in a position where the majority of its costs are fixed and on longterm contracts with vendors and service providers. The organization is then unable to reduce or remove further costs if the demand for IT reduces, unless it pays significant penalties to exit those deals. This can also restrain the ability of the organization to repeatedly increase capacity. Therefore, sourcing IT on a variable basis can form a good platform for continual optimization.

By adopting agility as a key principle for optimization, the organization will evaluate initiatives in a way that ranks those initiatives, keeping the costs agile at the top. Once the initiatives are implemented, the focus then moves to demand management - ensuring IT leads the business to consume only what is necessary, and providing options to consume IT in a way that steers the business down a consistent and optimized path.

\subsubsection{Accountability}

In order for IT to be fully optimized, both the supply and demand side must be reviewed. Too often, organizations see optimization as how to best supply the demand for IT. This can create a culture where IT becomes reactive to the business needs and not proactive enough to be able to influence 
demand. In order for IT to be continually optimized, leaders must take ownership of the futures of their departments. At a minimum, leaders must engage with the business to manage the capacity, features and functions that IT provides. If IT leaders can better predict, with some degree of certainty, the demands on IT, they will be in a strong position to source IT in the most optimal way. One strategy to make the business more aware of its demand for IT is to use techniques such as chargeback and show back.

\subsubsection{Simplification}

In terms of cost optimization, simplification is about reducing complexity in order to reduce costs. IT systems are inherently complex, but a lack of standard platforms, inconsistent business processes, and poorly defined IT services and service levels are more likely to drive up IT costs.

To be sure, there are factors other than complexity that drive high IT costs (for example, quality of IT staff, quality of leadership and geographic location). However, complexity does impose an unnecessary surcharge, and Gartner estimates that surcharge can be as much as 25 percent on a unit cost basis. That means the same IT unit of work could cost up to 25 percent more in a highly complex environment than it would in a streamlined environment.

However, the goal should never be to simplify at the expense of business value. Rather, organizations that are mature in terms of IT cost optimization will have identified and eliminated unnecessary levels of complexity. These unnecessary levels of complexity are areas where complexity adds no value to the enterprise.

\subsubsection{Discipline}

Organizations that are able to demonstrate continuous improvement and deliver on the initiatives planned see cost optimization as a discipline and not as a one-off or discrete project. The principle of discipline ensures that cost optimization has an accountable owner and is led from the top (normally by the CIO). It is essential to proactively manage cost optimization and think of longer-term cost targets, with dashboards and metrics to measure improvement, instead of waiting for targets to be handed down from the top management. Organizations that do this well often create a team including professionals from outside of IT to ensure optimization solutions consider business outcomes and constraints rather than purely technical specifications.

\subsection{IT Cost Optimization Forces you to Wrestle With Three Key Challenges:}

\subsubsection{Governance}

Are you running your IT organization like a business? If you're not, you should be. You need to control IT costs and transform your business at the same time. So, where are you investing for growth?

\subsubsection{IT Budgets}

Budget pressures continue in year by year. Is there a right way to cut costs? You need to deliver more productivity with fewer resources and provide more cost savings. What savings will your organization deliver?

\subsubsection{IT Cost Transparency}

You need to improve your level of IT transparency, yet too much transparency will cause needless questioning. How much is enough?

\section{RECOMMENDATIONS}

- Use the decision framework described here to prioritize cost optimization ideas.

- After prioritizing ideas, map them to a grid; help determine the level of effort the business leadership is committed to supporting. This graphic representation will help leaders fully appreciate the effort required and the relative benefits of each initiative.

- Execute well around cost optimization efforts. Do the small things quickly and deliver results to prove that IT can contribute to efficiency improvement.

- $\quad$ Get the CFO and budget analysts engaged in the process of identifying and prioritizing cost optimization ideas.

\section{CONCLUSIONS}

To build credibility, IT leaders need to carefully consider the kind of IT and business cost optimization they would propose. Business leadership must weigh in on the trade-offs of some reduction versus others. While capable of making these decisions, CIOs should avoid unilateral decision making. Unilateral decisions invariably result in a negative view of IR or of IT leadership, and also miss the point that IT exists to supports the business. Therefore, these decisions need to be made at the enterprise level.

IT leaders can improve the outcome of the cost optimization initiatives by expanding their analysis of these ideas to include the elements such as impact on the business, risk and level of investment required. Broadening the analysis and including those ideas in a broader portfolio management context with the organization IT governance process will streamline the process and improve outcomes 


\section{REFERENCES}

[1] http://www.archstoneconsulting.com/services/itstrategy-opeations/white-papers/optimizing-ittechnology.jsp

[2] http://www.cioinsight.com/it-news-trends/continuousit-cost-optimization-takes-center-stage.html

[3] http://www.itbusinessedge.com/slideshows/five-keyprinciples-for-continuous-it-cost-optimization-06.html

[4] http://www.cognizant.com/InsightsWhitepapers/IT_Co st_Optimization.pdf

[5] http://eval.symantec.com/mktginfo/enterprise/other_res ources/b-

gartner_decision_framework_for_prioritizing_cost_opt imization_ideas.en-us.pdf 